University of Nebraska - Lincoln

DigitalCommons@University of Nebraska - Lincoln

Faculty Publications from the Department of Electrical \& Computer Engineering, Department Electrical and Computer Engineering

2009

\title{
Missing-Sensor-Fault-Tolerant Control for SSSC FACTS Device With Real-Time Implementation
}

Wei Qiao

University of Nebraska-Lincoln, wqiao@engr.unl.edu

Ganesh Kumar Venayagamoorthy

Missouri University of Science and Technology, gkumar@ieee.org

Ronald G. Harley

Georgia Institute of Technology

Follow this and additional works at: https://digitalcommons.unl.edu/electricalengineeringfacpub

Part of the Electrical and Computer Engineering Commons

Qiao, Wei; Venayagamoorthy, Ganesh Kumar; and Harley, Ronald G., "Missing-Sensor-Fault-Tolerant Control for SSSC FACTS Device With Real-Time Implementation" (2009). Faculty Publications from the Department of Electrical and Computer Engineering. 135.

https://digitalcommons.unl.edu/electricalengineeringfacpub/135

This Article is brought to you for free and open access by the Electrical \& Computer Engineering, Department of at DigitalCommons@University of Nebraska - Lincoln. It has been accepted for inclusion in Faculty Publications from the Department of Electrical and Computer Engineering by an authorized administrator of DigitalCommons@University of Nebraska - Lincoln. 


\title{
Missing-Sensor-Fault-Tolerant Control for SSSC FACTS Device With Real-Time Implementation
}

\author{
Wei Qiao, Member, IEEE, Ganesh Kumar Venayagamoorthy, Senior Member, IEEE, and \\ Ronald G. Harley, Fellow, IEEE
}

\begin{abstract}
Control of power systems relies on the availability and quality of sensor measurements. However, measurements are inevitably subjected to faults caused by sensor failure, broken or bad connections, bad communication, or malfunction of some hardware or software. These faults, in turn, may cause the failure of power system controllers and consequently, severe contingencies in the power system. To avoid such contingencies, this paper presents a sensor evaluation and (missing sensor) restoration scheme (SERS) by using auto-associative neural networks (auto encoders) and particle swarm optimization. Based on the SERS, a missing-sensor-fault-tolerant control is developed for controlling a static synchronous series compensator (SSSC) connected to a power network. This missing-sensor fault-tolerant control (MSFTC) improves the reliability, maintainability, and survivability of the SSSC and the power network. The effectiveness of the MSFTC is demonstrated by a real-time implementation of an SSSC connected to the IEEE 10-machine 39-bus system on a Real Time Digital Simulator and TMS320C6701 digital signal processor platform. The proposed fault-tolerant control can be readily applied to many existing controllers in power systems.
\end{abstract}

Index Terms-Auto-associative neural network, fault-tolerant control, flexible ac transmission system (FACTS) device, missing sensor restoration, particle swarm optimization (PSO), real-time implementation, static synchronous series compensator (SSSC).

\section{INTRODUCTION}

C ONTROL of any plant (device or subsystem) in an electric power system depends on the availability and quality of sensor measurements. Measurements, however, can be corrupted or interrupted due to sensor failure, broken or bad connections, bad communication, or malfunction of some hardware or software (all of these are referred to as missing sensor measurements in this paper). If some sensors are missing, the controllers cannot provide the correct control actions for a plant

Manuscript received January 25, 2008; revised July 16, 2008. Current version published March 25, 2009. This work was supported by the National Science Foundation under Grant ECS \# 0524183. Paper no. TPWRD-00031-2008.

W. Qiao is with the Department of Electrical Engineering, University of $\mathrm{Ne}-$ braska-Lincoln, Lincoln, NE 68588-0511 USA (e-mail: wqiao@engr.unl.edu).

G. K. Venayagamoorthy is with the Real-Time Power and Intelligent Systems Laboratory, Department of Electrical and Computer Engineering, Missouri University of Science and Technology, Rolla, MO 65409-0249 USA (e-mail: gkumar@ieee.org).

R. G Harley is with the Intelligent Power Infrastructure Consortium (IPIC) in the School of Electrical and Computer Engineering, Georgia Institute of Technology, Atlanta, GA 30332-0250 USA (e-mail: rharley@ece.gatech.edu).

Color versions of one or more of the figures in this paper are available online at http://ieeexplore.ieee.org.

Digital Object Identifier 10.1109/TPWRD.2009.2016258 based on faulty input data. As a result, the plant may have to be tripped off from the power system.

In a power system, there are many devices (e.g., flexible ac transmission system (FACTS) devices) that play a crucial role in system regulation, control, and stability. For instance, the static synchronous series compensator (SSSC) [1]-[4], which is a voltage-source converter (VSC)-based FACTS device, is typically placed in series on a critical transmission line of the power network for power-flow control and power oscillation damping [3], [4]. Unexpected tripping of such an SSSC due to missing sensor measurements may cause overloading of some transmission lines and reduce the operating margin of the power system. If a severe disturbance occurs under this condition, further contingencies may occur and the system may lose stability. To avoid such contingencies, fault tolerance is an essential requirement for power system control.

A fault-tolerant control system [5] should be able to mitigate the effects of the sensor faults to an acceptable level by detecting and restoring the missing sensor data. This will improve the reliability, maintainability, and survivability of the power system. In principle, to achieve fault-tolerance, system redundancy is necessary. For many systems, certain degrees of redundancy are present among the data from various sensors. If the degree of redundancy is sufficiently high, the readings from one or more missing sensors may be able to be accurately restored from the remaining healthy sensor data.

State estimation [6] is commonly used to identify state variables that are not accessible from direct measurements, and could therefore be modified to restore missing sensor data. This technique is based on an analysis of the system model and the redundancy of system state variables. By deriving closed-form solutions for the variables corresponding to the missing sensors, the lost data are explicitly reconstructed from the remaining available data. However, for many systems, this model-based method converges slowly and the closed-form solutions can be unfeasible. Moreover, accurate system models are usually unavailable in real system applications.

In their previous work [7]-[9], the authors proposed and demonstrated several missing-sensor-fault-tolerant identification and control schemes for an SSSC by using auto-associative neural networks (auto-encoders) [10], [11] and particle swarm optimization (PSO) [12], [13]. These fault-tolerant schemes were demonstrated by simulation studies in PSCAD/EMTDC on a single-machine infinite bus (SMIB) [7], [8] or a small multimachine power system [9]. However, two important issues related to the practical system applications were not considered. First, these fault-tolerant schemes employ a PSO-based 
iterative search algorithm for online missing sensor restoration. Compared to the normal sampling frequency when no sensors are missing, the restored missing sensor data can only be sent to the SSSC controllers at a reduced sampling frequency in order to provide a sufficient period of time step to perform this online search algorithm. The use of a reduced sampling frequency may have a significant effect on the controller performance. This issue can only be investigated by real-time or hardware implementations. Second, practical power systems are large scale with complex dynamics. Therefore, the previous studies using software simulations on an SMIB or a small multimachine system do not sufficiently demonstrate the effectiveness of these fault-tolerant schemes in a practical power system.

This paper extends the work in [7]-[9] on a missing-sensorfault-tolerant control (MSFTC) for controlling an SSSC in a power network. The MSFTC contains a conventional internal and external control scheme [2], [3] (i.e., without any fault-tolerant design) cascaded with a sensor evaluation and (missing sensor) restoration scheme (SERS). The conventional control scheme provides the correct control actions for the SSSC under the condition that all the required sensor data are available. The SERS is designed by using the auto-encoders and PSO. It evaluates the integrity of the sensor data used by the SSSC controllers. If some sensor data are missing, it is able to detect and restore the missing sensor data and, therefore, provides a set of complete sensor measurements to the SSSC controllers. The resulting MSFTC therefore provides a missing-senor-fault-tolerant control for the SSSC. The proposed MSFTC is validated by a real-time implementation on an SSSC connected to the IEEE 10-machine 39-bus system, using a Real-Time Digital Simulator (RTDS) and TMS320C6701 digital signal processor (DSP) platform.

\section{SSSC AND ITS CONVENTIONAL CONTROL}

The SSSC in Fig. 1 is a VSC-based series FACTS device which injects a controllable voltage in quadrature with the line current of a power network. This is equivalent to rapidly providing a controllable capacitive or inductive reactance compensation independent of the line current [2]. Moreover, with a suitably designed external damping controller [3], [4], the SSSC can also be used to improve damping of the low-frequency transient power oscillations in a power network. These features make the SSSC an attractive FACTS device for power-flow control, power oscillation damping, and improving transient stability. This section briefly discusses the conventional internal and external control of the SSSC.

The main objectives of the SSSC internal control [2] (Fig. 1) are to inject a controllable voltage $v_{c, a b c}$ (by injecting a desired compensating reactance $X_{C}^{*}$ ) at the ac terminals of the inverter as well as to keep the dc terminal voltage of the inverter constant at steady state.

The objective of the SSSC external control (Fig. 2) is to damp transient power oscillations of the system. This external controller is able to rapidly change the compensating reactance $X_{\mathrm{C}}$ injected by the SSSC, thus providing supplementary damping during transient power swings [3], [4]. In a practical controller, it is usually desirable to choose a local signal. In this paper, the active power deviation $\Delta P_{L}$ on the transmission line, measured

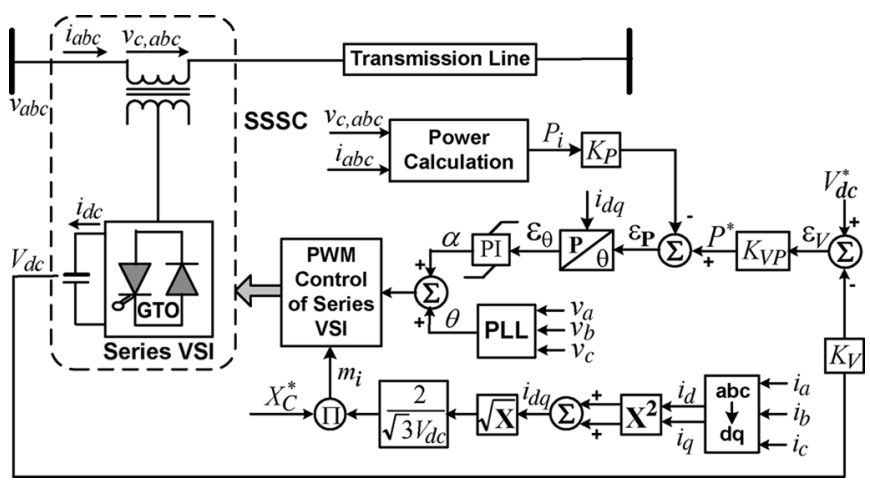

Fig. 1. Schematic diagram of the SSSC internal controller.

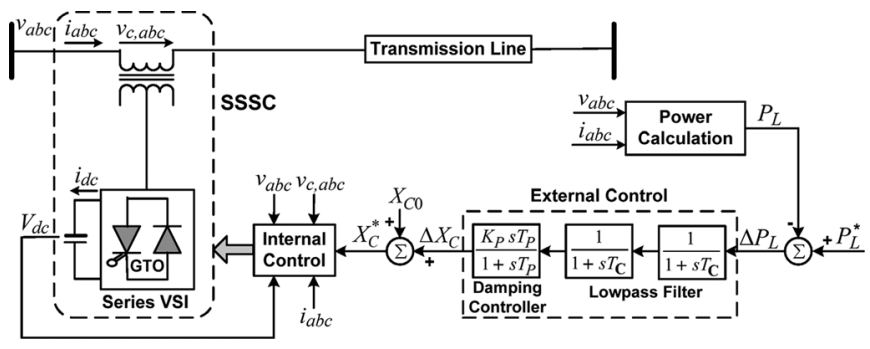

Fig. 2. Schematic diagram of the SSSC external damping controller.

at the connection point of the SSSC, is used as the input signal to the external controller. In Fig. 2, $\Delta P_{L}$ is passed through two first-order low-pass filters and a damping controller (consisting of a proportional damping gain $K_{P}$ and a washout filter) to form a supplementary control signal $\Delta X_{C}$, which is then added to a steady-state fixed set-point value $X_{C 0}$ to form the total commanded value of compensating reactance $X_{C}^{*}$ at the input of the SSSC internal controller [9]. The washout filter is a high-pass filter that removes the dc offset, and without it, the steady changes in active power $P_{L}$ would modify the value of compensating reactance. The use of two low-pass filters is based on two reasons: 1) filtering the electrical noise in the measurements and 2) phase compensation to ensure that the variations in compensating reactance are correctly phased with respect to the transient power oscillations in order to provide supplementary damping.

\section{Missing Sensor Restoration AlgORithm}

An auto-encoder can learn the data correlations through inspection of historical data. Once trained, data correlations established by the auto-encoder can be used by some search algorithms (e.g., PSO in this paper) to restore missing data if the data dependency is sufficiently strong. This completes a missing sensor restoration algorithm (MSR), as shown in Fig. 3. The unique point of convergence of the MSR rests on the concepts of contractive and nonexpansive mappings [14] and the operating point determined by the healthy sensor data.

\section{A. Auto-Encoder [Fig. 3(a)]}

The auto-encoder [7]-[9] is a three-layer feedforward neural network with sigmoidal nonlinearity in the hidden layer. It is trained to perform an identity mapping, where the network inputs are reproduced at the output layer. The network contains a 


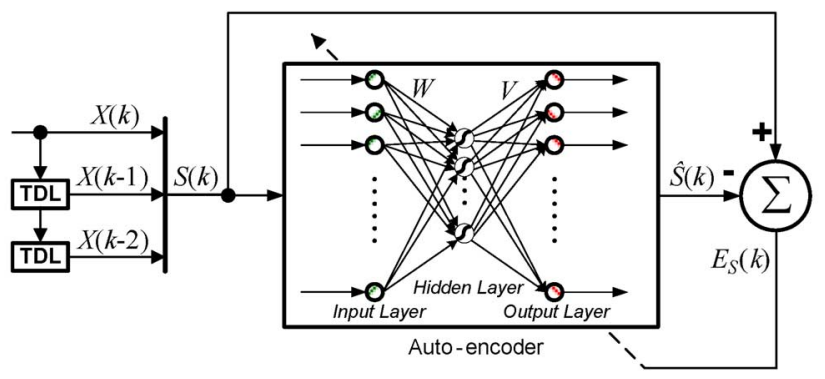

(a)

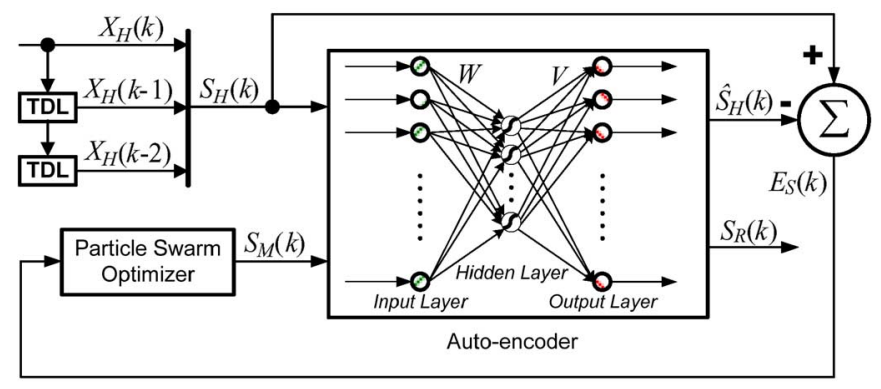

(b)

Fig. 3. Overall structure of the MSR: TDL denotes time delay lock. (a) Training of the auto-encoder. (b) Online restoration of missing sensor data.

hidden "bottleneck" layer which has fewer nodes than the input and output layers. The dimensionality reduction through the input-to-hidden layer enables the network to extract significant features, without restriction on the character of the nonlinearities in the data (nonlinear feature extraction). Hence, the hidden layer captures the correlations between all input data. On the other hand, the dimensionality expansion through the hidden-tooutput layer enables the network to reproduce the high dimensional inputs at the output layer. The overall input-output mapping for the auto-encoder $\hat{g}: S \in R^{P} \rightarrow \hat{S} \in R^{P}$ is

$$
\hat{S}_{i}=\hat{g}\left(S, W, V_{i}\right)=V_{i} \cdot d(S, W)=\sum_{j=1}^{q} V_{i j} d_{j}\left(S, W_{j}\right)
$$

where $i=1,2, \cdots, p ; p$ is the dimension of the input and output vectors; $q$ is the number of hidden-layer neurons; $S$ is the input vector; $\hat{S}_{i}$ is the $i$ th output; $W$ and $V$ are the input and output weight matrices, respectively; and $d_{j}\left(S, W_{j}\right)$ is the sigmoid activation function of the $j^{\text {th }}$ hidden-layer neuron, given by

$$
d_{j}\left(S, W_{j}\right)=\frac{1}{1+e^{-a_{j}}}
$$

where

$$
a_{j}=W_{j} \cdot S=\sum_{i=1}^{p} W_{j i} S_{i}
$$

Suppose the vector $X=\left[x_{1}, x_{2}, \cdots, x_{n}\right]$ consists of the measured time-varying sensor data at each time sampling $k$. In a power system, the time-varying variables are generally periodic and in the sinusoidal form, given by

$$
x_{i}(k)=A_{i} \sin \left(\omega_{i} k+\phi_{i}\right) \quad i=1, \cdots, n .
$$

Each periodic time-varying variable $x_{i}(k)$ is auto-correlated and its feature is determined by the magnitude $A_{i}$, the angular frequency $\omega_{i}$, and the phase angle $\phi_{i}$. Auto-correlations can be used to extract the significant features buried in a periodic time-varying signal and, therefore, are useful to restore the missing time-varying sensor measurements. The auto-correlation of each variable in the vector $X$ can be captured by the auto-encoder using the time-delayed inputs. As shown in Fig. 3(a), the inputs of the auto-encoder $S$ consist of the vector $X$ at the present time step as well as at the previous two time steps (i.e., $S(k)=[X(k), X(k-1), X(k-2)])$.

The auto-encoder is first trained without any missing sensor. It starts off with small random initial weights. By feeding the data through the auto-encoder and adjusting its weight matrices (using the backpropagation algorithm [15]) $W$ and $V$, the autoencoder is trained to reproduce its input data at its output layer. Once trained, the cross correlations between different sensor data as well as the auto-correlations of each sensor data in the vector $X$ are established by the auto-encoder. The auto-encoder with fixed parameters is then used for sensor evaluation and missing sensor restoration.

\section{B. Missing Sensor Restoration [Fig. 3(b)]}

After training, the auto-encoder with fixed parameters is able to reproduce its inputs at its output layer. Under such a condition, if one or more sensor measurements are missing, the outputs of the auto-encoder $\hat{S}$ no longer match its inputs $S$, and the error signal $E_{S}$ in Fig. 3(a) becomes significant. In this case, the PSO module in the feedback search loop of the MSR is activated and only the healthy sensor data $S_{H}$ are fed directly into the auto-encoder. The error signal $E_{S}$ is then used by the PSO as a fitness signal to search for the optimal estimates of the missing sensor data, based on the correlations between the healthy data and the missing data, established by the auto-encoder. At each iteration, the estimated missing sensor data $S_{M}$ by the PSO, which is the swarm best position that the PSO achieves so far, is fed together with the healthy sensor data through the auto-encoder to reduce the value of the following fitness measure function $f$ for each particle, defined by:

$$
f=\left\|E_{S}\right\|=\left\|S_{H}-\hat{S}_{H}\left(S_{H}, S_{M}\right)\right\|
$$

where $S_{H}$ represents the actual healthy sensor data and $\hat{S}_{H}$ represents the reproduced healthy sensor data from the auto-encoder. Once the error $\left\|E_{S}\right\|$ is below a predetermined threshold, the output of the auto-encoder $S_{R}$ is regarded as the best estimates of the missing sensor data.

The use of the auto-encoder does not need an explicit plant model. In addition, the PSO algorithm provides a fast and efficient search for the optimal solution, because of its attractive features including simple implementation, small computational load, and fast convergence. Therefore, the MSR algorithm is suitable for online applications.

\section{Convergence of the MSR}

The unique convergence of the MSR can be shown through the concepts of contractive and nonexpansive mapping. An operator $\Theta$ mapping $\Re^{N} \rightarrow \Re^{N}$ is contractive if, for any vectors, 
$x \in \Re^{N}$ and $y \in \Re^{N}$, it follows that $\|\Theta x-\Theta y\|<\|x-y\|$, where $\|\cdot\|$ denotes the Euclidean norm. The operator $\Theta$ is nonexpansive if $\|\Theta x-\Theta y\| \leq\|x-y\|$. According to the Banach Fixed-Point Theorem [16], if $\Theta$ is a contractive mapping, then a unique fixed point $x_{0}$ exists for which $\Theta x_{0}=x_{0}$; if $\Theta$ is nonexpansive, then a plurality of fixed points $x_{0}$ may exist for which $\Theta x_{0}=x_{0}$.

A well-trained auto-encoder constructs a nonexpansive mapping $\hat{g}$ between its input space $\underline{\mathrm{S}} \subset R^{P}$ and output space $\underline{\hat{\mathrm{S}}} \subset$ $R^{P}$, because for any vector $S \in \underline{\mathrm{S}}$, a unique vector $\hat{S} \in \underline{\hat{S}}$ exists so that $\hat{S}=\hat{g}(S)=S$. The auto-encoder performs a data compression through the input-to-hidden layer to extract significant features in the data. To avoid losing any significant information in the data, a necessary condition is that the dimension $q$ of the hidden layer must be equal to or exceed the number of degrees of freedom $n$ in the input data.

Suppose $r$ sensor data in $S$ are missing, then the number of healthy sensor data is $p-r$. If the degree of data redundancy is sufficiently high (i.e., the number of degrees of freedom of the $p-r$ healthy sensor data is still $n$ ), then the "operating point" defined by the set of healthy sensor data $S_{H}$ is the same as that defined by the full set of sensor data $S=\left[S_{H}, S_{M}\right]$, because the missing sensor data $S_{M}$ are redundant. Here, $S_{M}$ represents the correct values of the missing sensor data. Under such a condition, given an "operating point" defined by the set of healthy sensors, a unique point of convergence exists for a welltrained auto-encoder. This convergence point should be reached regardless of how the missing sensors are initialized. Generally, such a condition is satisfied if $(p-r)>q$ because $q \geq n$.

On the other hand, if the degree of data redundancy is not sufficiently high (i.e., $(p-r)<q)$, then there may be $(p-r)<$ $n$. Under such a condition, the number of degrees of freedom of the $p-r$ healthy sensor data is less than $n$. Therefore, different sets of missing sensor data $S_{M 1}$ and $S_{M 2}$ may exist so that $S_{1}=\hat{g}\left(S_{1}\right)$ and $S_{2}=\hat{g}\left(S_{2}\right)$, where $S_{1}=\left[S_{H}, S_{M 1}\right]$ and $S_{2}=\left[S_{H}, S_{M 2}\right]$. Here, $S_{M 1}$ and $S_{M 2}$ are the correct values of the missing sensor data. In other words, given the set of healthy sensor data $S_{H}$, the missing sensor data might be restored as different values by the MSR. To avoid such a result, a necessary condition for the auto-encoder to work is that the number of healthy inputs must be equal to or exceed the number of degrees of freedom in the hidden layer [11].

To conclude, generally with a well-trained auto-encoder, the unique convergence of the MSR is achieved if two conditions are satisfied: 1) $q>n$ and 2) $(p-r)>q$, and vice versa.

\section{Missing-Sensor-Fault-Tolerant Control}

Control of the SSSC (Figs. 1 and 2) relies on the availability and quality of four sets of sensor measurements: the three-phase currents $i_{a b c}$ of the transmission line, the three-phase bus voltages $v_{a b c}$, the injected three-phase voltages $v_{c, a b c}$ of the SSSC, and the dc-link voltage $V_{\mathrm{dc}}$. Other variables, such as $P_{i}$ and $P_{L}$, are calculated from these measured variables. In addition, the dc-link current $i_{\mathrm{dc}}$ is also measured to protect the inverter from overcurrent caused by the short-circuit fault of the dc link [17]. However, the value of $i_{\mathrm{dc}}$ is irrelevant to the performance of the SSSC controllers. In this section, the MSFTC is designed for

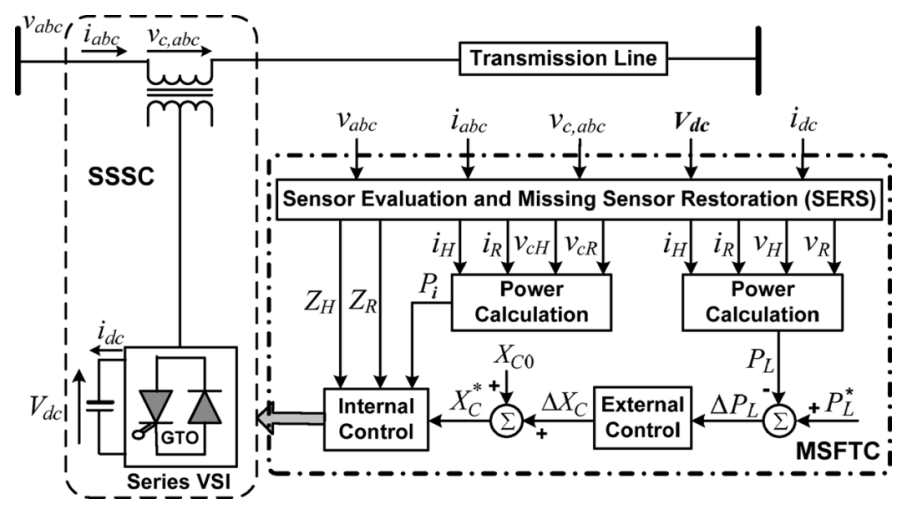

Fig. 4. Overall structure of the proposed missing-sensor-fault-tolerant control (MSFTC) strategy.

both internal and external control of the SSSC. This MSFTC provides fault tolerance to any set of major sensors $\left(i_{a b c}, v_{a b c}\right.$, $v_{c, a b c}$, and $V_{\mathrm{dc}}$ ) faults based on two reasonable assumptions: 1) there are no multiple sets of sensors missing and 2) the power system operates under a three-phase balanced condition at the transmission level.

\section{A. Overall Structure of the MSFTC}

Fig. 4 shows the overall structure of the MSFTC for the SSSC. It consists of an internal controller, an external controller, and an SERS. The four sets of sensor data used by the SSSC internal and external controllers are fed into the SERS, which evaluates the integrity of these sensor data. If the SERS detects that one or more sensors are missing, it is responsible for restoring all of the missing sensor data. The output variables of the SERS with a subscript $R$ represent the restored missing sensor data, while the output variables with a subscript $H$ represent the healthy sensor data. If there is no sensor missing, the outputs with a subscript $H$ are exactly the same as the corresponding inputs (e.g., $i_{H}=i_{a b c}$ ). The active power $P_{L}$ used by the external controller is calculated from $\left[i_{H}, i_{R}\right]$ and $\left[v_{H}, v_{R}\right]$, and the active power $P_{i}$ used by the internal controller is calculated from $\left[i_{H}, i_{R}\right]$ and $\left[v_{c H}, v_{c R}\right]$. Other sensor data used by the internal controller consist of $Z_{H}=\left[i_{H}, v_{H}, V_{d c H}\right]$ and $Z_{R}=\left[i_{R}, v_{R}, V_{d c R}\right]$. The SERS provides a set of complete sensor data to the SSSC controllers even when some sensors are missing and, therefore, guarantees a fault-tolerant control strategy for the SSSC.

\section{B. Design of the SERS}

1) Three-Phase Current Sensor Measurements: Power systems normally operate under almost balanced three-phase conditions at the transmission level. Thus, the three-phase currents $i_{a}, i_{b}$, and $i_{c}$ should approximately satisfy the following equation:

$$
i_{a}+i_{b}+i_{c}=0 .
$$

A more realistic expression for (6) can be written as

$$
\left|i_{a}+i_{b}+i_{c}\right|<\sigma_{1}
$$

where $\sigma_{1}$ is a predetermined small threshold. Under balanced conditions, if the aforementioned relationship (7) is not satis- 


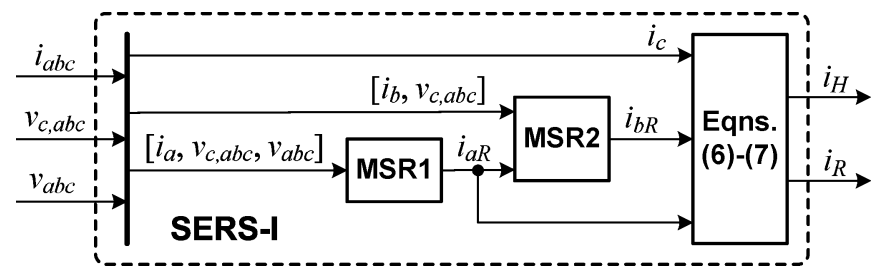

Fig. 5. Structure of the SERS-I.

fied, it indicates that one or more current sensors are missing. However, if $i_{a}, i_{b}$, and $i_{c}$ are all missing, there might be $i_{a}=$ $i_{b}=i_{c}=0$ and, therefore, (7) is still satisfied; to distinguish such a case from the case of no missing sensor, another equation is used, given by

$$
\left|i_{a}\right|<\sigma_{2} \text { and }\left|i_{b}\right|<\sigma_{2} \text { and }\left|i_{c}\right|<\sigma_{2}
$$

where $\sigma_{2}$ is a predetermined small threshold. If (7) is satisfied but (8) is not satisfied, there is no sensor missing. Otherwise, one or more phase current sensors are missing.

If only one phase current sensor is missing, it can simply be restored by using (6). However, in order to detect and restore multiple missing current sensors, a sensor evaluation and missing sensor restoration scheme (SERS-I) is designed, as shown in Fig. 5. A necessary condition for SERS-I implementation is that all the sensor data in $v_{c, a b c}$ and $v_{a b c}$ are available. How to determine this condition is discussed later in Section IV-B3) on the overall structure of the SERS. Here, it is simply assumed that this condition is satisfied. The SERS-I contains two MSR blocks and a block that implements (6) and (7). Each MSR block has the same structure as shown in Fig. 3 and only evaluates the status of one current sensor. If any MSR block detects that the current sensor is missing, its PSO module is then activated and only performs a 1-D search to restore the missing current. In Fig. 5, $i_{a}$ is evaluated by MSR1. If $i_{a}$ is missing, it is restored by MSR 1 and the restored value $i_{a R}$ is then used as the healthy input for MSR2 and the block (6), (7); if $i_{a}$ is healthy, then $i_{a R}=i_{a}$. In a similar way, $i_{b}$ is evaluated by MSR2. If $i_{b}$ is missing, it is restored by MSR2 and the restored value $i_{b R}$ is then used as the healthy input for the block (6), (7); if $i_{b}$ is healthy, then $i_{b R}=i_{b}$. $i_{c}$ is evaluated by (7). If $i_{c}$ is missing, it is calculated by (6). The output vector $i_{R}$ of the SERS-I contains the total restored missing current sensor data; but $i_{H}$ contains other healthy current sensor data. These two vectors provide a set of complete current sensor measurements to the SSSC controllers. In this design, MSR1 needs to use the other six sensors' data $v_{c, a b c}$ and $v_{a b c}$ in order to provide the required redundancy to restore the missing current $i_{a}$. However, the four sensors' data $v_{c, a b c}$ and $i_{a R}$ are enough to provide MSR2 with the required redundancy to restore the missing current $i_{b}$.

The implementation procedure of the SERS-I is shown as a flowchart in Fig. 6, where $\varepsilon_{1}$ and $\varepsilon_{2}$ are predetermined small thresholds for MSR1 and MSR2, respectively. If the error signal, for example, $\left\|E_{s 1}\right\|$ of MSR1 (Fig. 3) is smaller than the threshold $\varepsilon_{1}$, it indicates that $i_{a}$, which is monitored by MSR1, is healthy; otherwise, if $\left\|E_{s 1}\right\|>\varepsilon_{1}$, it indicates that $i_{a}$ is missing and is restored by MSR1. Similarly, if $\left\|E_{s 2}\right\|<\varepsilon_{2}$,

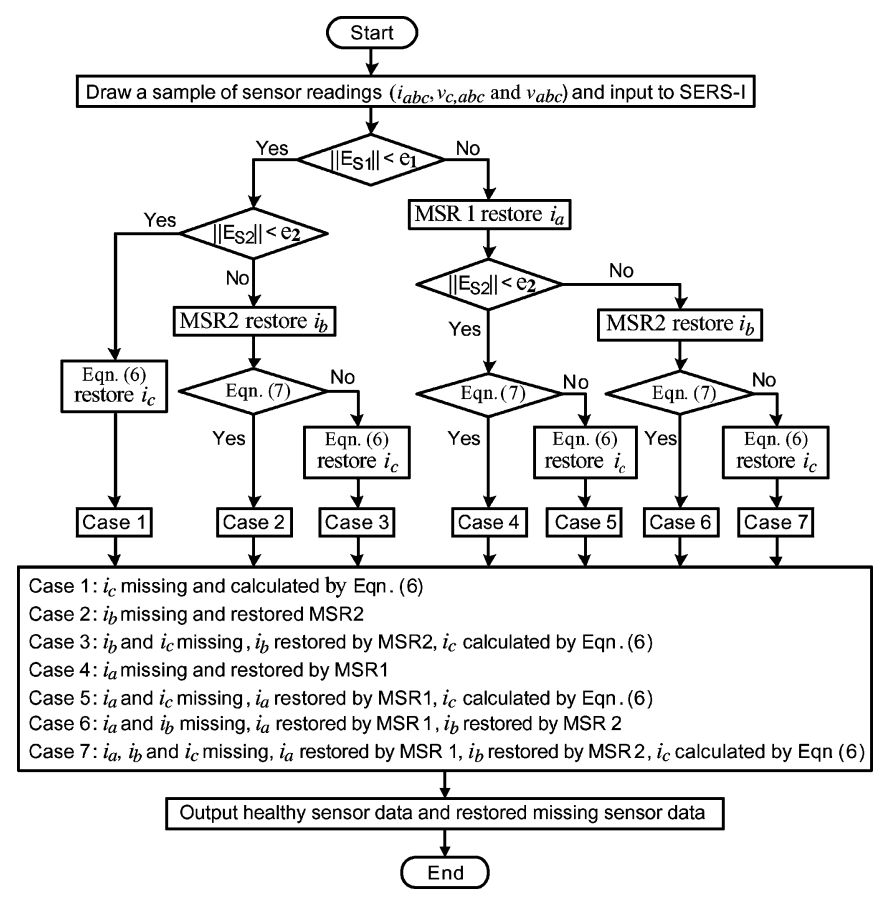

Fig. 6. Implementation procedure of the SERS-I.

then $i_{b}$ is healthy; otherwise, if $\left\|E_{s 2}\right\|>\varepsilon_{2}$, then $i_{b}$ is missing and is restored by MSR2.

The use of the cascading structure to design the SERS-I is based on the following reasons: 1) this structure enables the SERS-I itself to evaluate the status of the sensor data and detect which sensor or sensors are missing; 2) each MSR only searches in a 1-D space to restore one missing sensor measurement, which is faster than only using one MSR to search in a multidimensional space in order to restore multiple missing sensor measurements; and 3) the required degree of data redundancy for restoring one missing sensor is lower than that of restoring multiple missing sensors for each MSR and, therefore, fewer sensor data need to be used.

In this application, a small population of particles (five particles) is used in PSO to reduce the computational cost of the PSO search algorithm. Each MSR converges within 15 iterations to restore one missing sensor measurement. Therefore, the maximum iteration number for the PSO implementation in each MSR block is set at $M=15$. In addition, a necessary condition for the MSR to work is that the number of healthy inputs of the auto-encoder must be equal or exceed the number of degrees of freedom in its hidden layer. Thus, the dimensions of the input, hidden, and output layers of the auto-encoders in MSR1 and MSR2 are chosen to be $21 \times 12 \times 21$ and $15 \times 10 \times 15$, respectively.

2) DC-Link Voltage Sensor: Under normal operating conditions, the dc-link voltage is almost constant and its value is far from zero. The following power balance should be held while taking into account the dynamics of the dc-link:

$$
E=\left|P_{i}-P_{\text {loss }}-C V_{d c} \frac{d V_{d c}}{d t}\right|
$$

where $P_{i}$ is the measured active power injected to the SSSC (Fig. 1); $P_{\text {loss }}$ denotes the estimated power losses, including the 


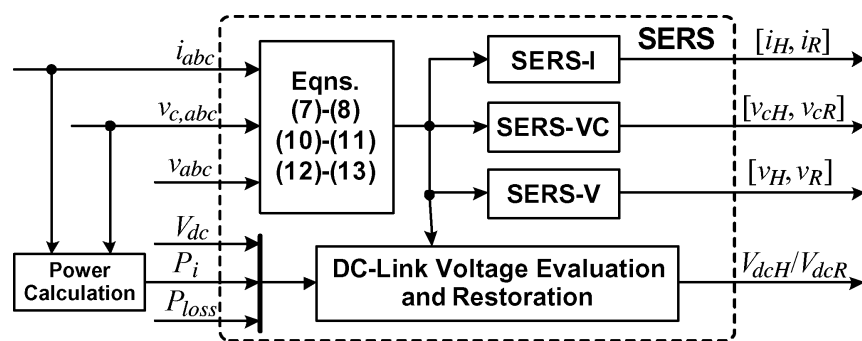

Fig. 7. Overall structure of the SERS

copper loss, iron loss, switching loss, etc., in the SSSC. A simplified method to estimate the power losses in a three-phase converter has been discussed in [18]. In practice, if $E>\sigma_{3}$, where $\sigma_{3}$ is a predetermined threshhold, then the measured dc-link voltage is replaced by the calculated value by using $V_{\mathrm{dc}}=$ $\int i_{\mathrm{dc}} \mathrm{dt} / C$.

3) Overall Structure of the SERS: Fig. 7 shows the overall structure of the SERS. The structure and implementation of the SERS-I block have been shown in Figs. 5 and 6, respectively. The SERS-VC and SERS-V blocks, which have the same structures as the SERS-I block, are used to evaluate the sensor data and restore the missing sensor data in $v_{c, a b c}$ and $v_{a b c}$, respectively. The status of the sensor data in $i_{a b c}, v_{c, a b c}$, and $v_{a b c}$ is preevaluated by the equation evaluation block called (7), (8), (10-(13), where (10)-(13) are given by

$$
\begin{aligned}
& \left|v_{c a}+v_{c b}+v_{c c}\right|<\sigma_{4} \\
& \left|v_{c a}\right|<\sigma_{5} \text { and }\left|v_{c b}\right|<\sigma_{5} \text { and }\left|v_{c c}\right|<\sigma_{5} \\
& \left|v_{a}+v_{b}+v_{c}\right|<\sigma_{6} \\
& \left|v_{a}\right|<\sigma_{7} \text { and }\left|v_{b}\right|<\sigma_{7} \text { and }\left|v_{c}\right|<\sigma_{7}
\end{aligned}
$$

where $\sigma_{4}, \sigma_{5}, \sigma_{6}$, and $\sigma_{7}$ are small threshholds. If (10) is satisfied but (11) is not satisfied, there is no sensor missing in $v_{c, a b c}$; otherwise, one or more sensors in $v_{c, a b c}$ are missing. If (12) is satisfied but (13) is not satisfied, there is no sensor missing in $v_{a b c}$; otherwise, one or more sensors in $v_{a b c}$ are missing. If the equation evaluation block detects that any of the three vectors $i_{a b c}, v_{c, a b c}$, and $v_{a b c}$ contain missing sensor data, it will activate the corresponding SERS-X (X represents I, VC, or V) block to identify and restore the missing sensors. The healthy sensor data and restored missing sensor data are then output from the SERS. If there is no missing sensor, the SERS outputs all of the healthy sensor data. This procedure is shown in the flowchart of Fig. 8 .

4) Training of the Auto-Encoders: The auto-encoders in the two MSR blocks of each SERS-X are continuously trained online simultaneously without any missing sensors (determined by (7), (8), and (10)-(13)). By feeding forward the data through the auto-encoder and adjusting its weight matrices $W$ and $V$, using the backpropagation algorithm, the auto-encoder is trained to map its inputs to its outputs as shown in Fig. 3(a). After training for every $N_{T}$ time step, the weights of each auto-encoder are frozen for $N_{E}$ time steps to evaluate the convergence of the auto-encoder. During the evaluation, if the error $\left\|E_{S}\right\|$ of each auto-encoder is beyond a specified threshold $\mu$ at any evaluation time step, the training resumes for the next $N_{T}$ time steps. Otherwise, if the error $\left\|E_{S}\right\|$ of each auto-encoder is below the threshold $\mu$ during the entire $N_{E}$ time step, the training stops

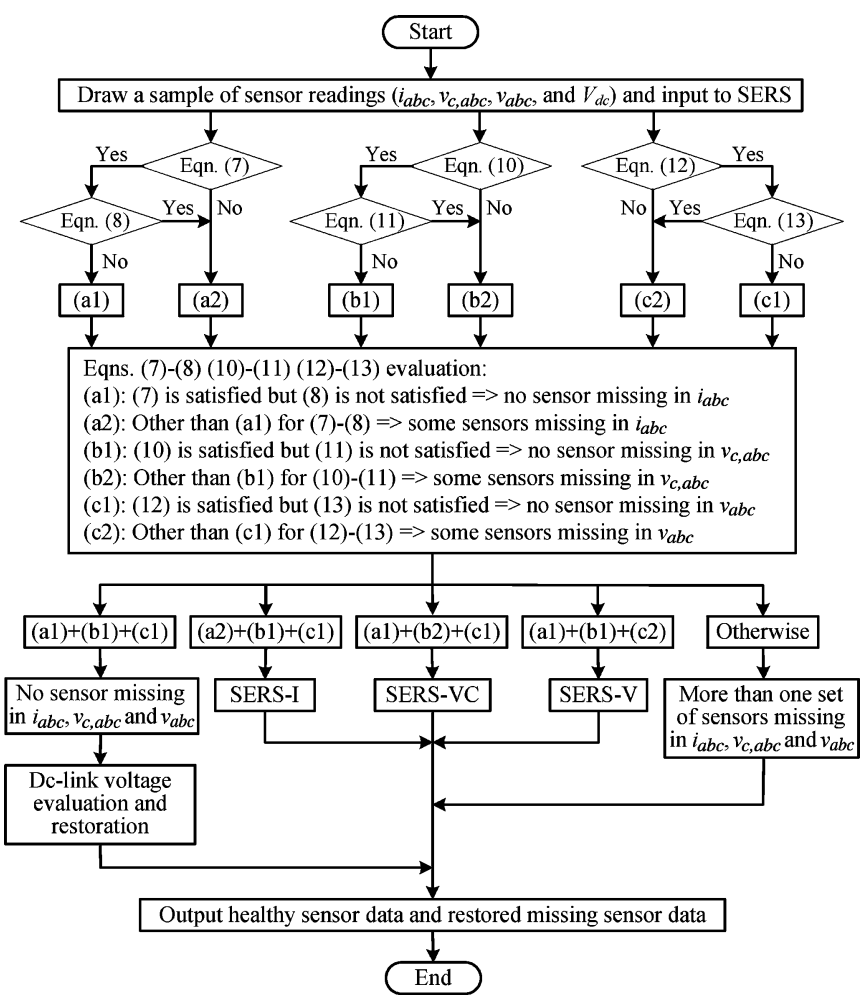

Fig. 8. Implementation procedure of the SERS.

and the auto-encoder is used for sensor evaluation and missing sensor restoration. If the system changes to a new operating point, the error $\left\|E_{S}\right\|$ might be beyond the threshold $\mu$ again. In this case, if there is no sensor missing, the training resumes to adapt to this new operating point.

\section{Unbalanced Operation}

The transmission system of a power network normally operates under a nearly balanced three-phase condition. The unbalanced operations are mainly caused by grid disturbances, such as unbalanced faults including a single-phase-to-ground fault, phase-to-phase fault, etc. Under these conditions, the transmission system experiences a short-term unbalanced operation (e.g., typically 50-200 ms) during the fault, and returns to its balanced three-phase operation after the fault is cleared. During the short-term unbalanced fault, (7), (8), and (10)-(13) are not applicable to evaluate the status of the sensor data; therefore, the equation evaluation block (7), (8) and (10)-(13) in Fig. 7 is temporarily deactivated, and the SERS continues to restore the missing sensors if they exist. In addition, if three sensor data in one set of sensor measurements (e.g., the three current sensors), are all missing during an unbalanced operation, then the third missing sensor cannot be accurately restored by (6). However, since the fault only exists for a very short period of time, it does not have any notable effect on the performance of the MSFTC.

The long-term unbalanced operations are mainly caused by an unbalanced load, transformers with different single phase units, untransposed transmission lines, etc. The effects of longterm unbalanced operations are normally small since the transmission system is still close to balanced operation [19]. 


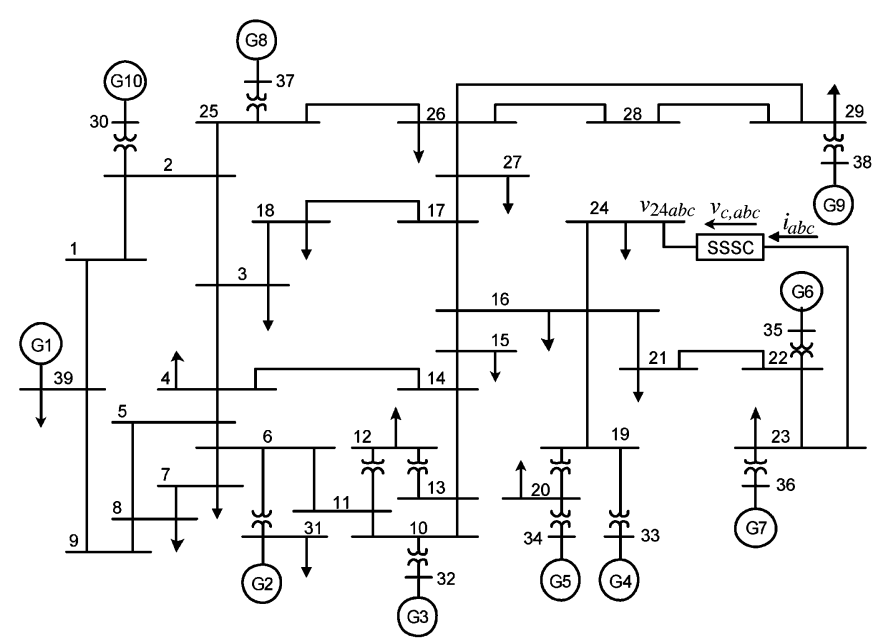

Fig. 9. Single-line diagram of an SSSC connected to the IEEE 10-machine 39-bus New England system.

\section{REAL-TIME IMPLEMENTATION PLATFORM}

To demonstrate the effectiveness of the MSFTC, the IEEE 10-machine 39-bus New England system [20], as shown in Fig. 9 , is now considered. An SSSC is connected to the bus 24 end of the transmission line 23-24 to regulate its power flow. This arrangement also improves the transient stability of this multimachine power system [21]. In this study, G10 is modeled as a three-phase infinite source, while the other nine synchronous generators (G1-G9) are modeled in detail, with the turbine governor and AVR/exciter dynamics taken into account [22]. The SSSC is represented by a detailed switching-level model, in which the PWM voltage source inverter is fully represented by individual GTO switches.

In this application, the operation and control of the SSSC rely on the availability and quality of four sets of sensor measurements: the three-phase currents $i_{a}, i_{b}, i_{c}$, of line 22-24; the injected three-phase voltages $v_{c a}, v_{c b}$, and $v_{c c}$ of the SSSC; the three-phase voltages $v_{24 a}, v_{24 b}$, and $v_{24 c}$ of bus 24; and the dc-link voltage $V_{\mathrm{dc}}$. The MSFTC as shown in Fig. 4 is applied to provide the fault-tolerant control for the SSSC.

The proposed MSFTC for the SSSC is validated by a realtime implementation by using an RTDS and an Innovative Integration M67 DSP card (based on a TMS320C6701-167 processor). Fig. 10 shows the real-time implementation platform. The SSSC and its internal and external controllers as well as the IEEE 39-bus power system are implemented on the RTDS; while the SERS is implemented on the M67 DSP card. This M67 DSP card communicates with the RTDS in real-time through a DSP-RTDS interface. The RTDS is equipped with analog input/ output channels [23]. The sensor data used by the SSSC controllers are sent through the RTDS analog output channels and DSP-RTDS interface to the M67 DSP card for the implementation of the SERS. The M67 DSP card, operating at $167 \mathrm{MHz}$, is hosted on a Pentium III $433 \mathrm{MHz}$ personal computer (PC) and equipped with two OMINBUS A4D4 I/O modules [24]. Each A4D4 module has four analog-to-digital (A/D) converters and four digital-to-analog (D/A) converters. The A4D4 modules receive the analog inputs (sensor data) from the DSP-RTDS in-

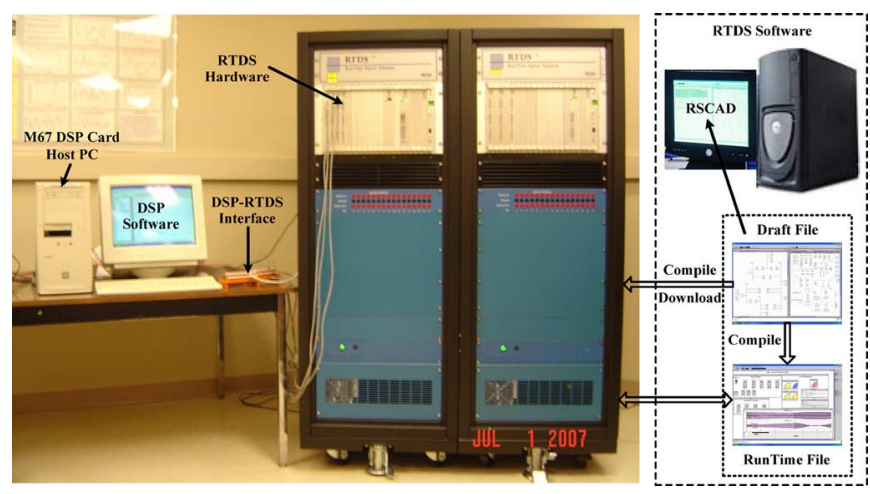

Fig. 10. Real-time implementation platform.

terface and convert them to digital signals. They are then used by the SERS, running on the TMS320C6701-167 processor, for sensor evaluation and missing sensor restoration. The healthy sensor data and the restored missing sensor data are sent back to the RTDS through the DSP-RTDS interface and RTDS analog input channels for fault-tolerant internal and external control of the SSSC.

If there is no missing sensor, the SERS only performs the equation evaluation block (7), (8), (10)-(13) to evaluate the integrity of the sensor measurements. Therefore, a high sampling rate, such as $5 \mathrm{kHz}$, can be used for the SERS implementation. However, if some sensors are missing, the SERS has to perform a PSO-based iterative search algorithm to restore the missing sensor data. In this case, the sampling rate for the SERS implementation is reduced to $50 \mathrm{~Hz}$ to ensure that the missing sensors can be restored within one sampling period of $20 \mathrm{~ms}$ using the MSR algorithm with the maximum iteration number of 15 . More discussions on choosing the sampling frequency are provided in Section VII.

\section{REAL-Time IMPLEMENTATION RESULTS}

The dynamic performance of the MSFTC is evaluated by applying different disturbances, such as unbalanced and balanced grid faults and change of load conditions, on the system in Fig. 9 that is implemented on the RTDS (Fig. 10). For each disturbance event, three (missing) sensor tests are performed and compared to evaluate the fault tolerance of the MSFTC: no sensor missing, two current sensors $i_{b}$ and $i_{c}$ missing, and three current sensors $i_{a}, i_{b}$, and $i_{c}$ missing. In a practical system, if some sensors are missing, their values may be read as zeros, some noise, or some uncertain error values. Since the forms of missing sensor readings have no effect on the implementation of the SERS during the real-time tests, the sensor readings are simply set to zeros if they are missing.

\section{A. Single-Phase-to-Ground (A-G) Fault}

The system in Fig. 9 is first operated at a normal operating condition without any missing sensors. The auto-encoder in each MSR block of the SERS is continuously trained online without any missing sensor, as discussed in Section IV. The online training of the auto-encoders converges within several minutes. Then, the training stops and the SERS continuously works online to evaluate the status of the sensor measurements 

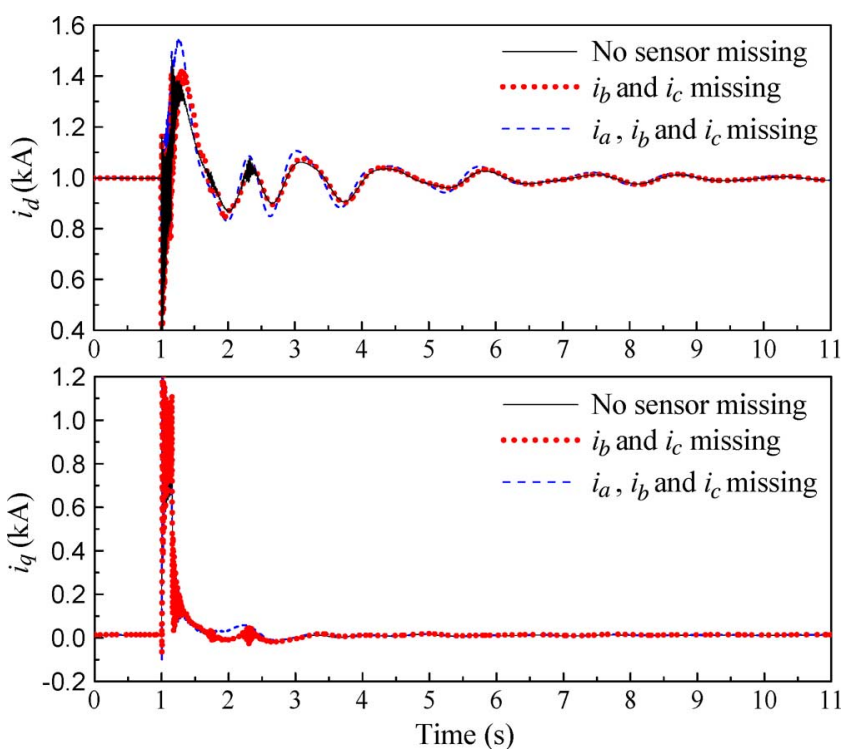

Fig. 11. Dynamic performance of the MSFTC with different (missing) sensor tests during a single-phase-to-ground (A-G) fault: $i_{d}$ and $i_{q}$.

and restore the missing sensor data. Thereafter, the first test is performed in which a temporary unbalanced phase A-to-ground short circuit is applied at the bus 22 end of the transmission line 21-22. The fault is cleared $150 \mathrm{~ms}$ after it has been applied. After the system returns to steady state, the second test is then performed in which two phase current sensors $i_{b}$ and $i_{c}$ are set to be missing. Thereafter, the same phase A-to-ground fault is applied to the system. Again, after the system returns to steady state, the third test is performed in which all of the three phase current sensors $i_{a}, i_{b}$, and $i_{c}$ are set to be missing and the same grid fault is applied to the system.

If some phase current sensors are missing, they are detected and restored by the SERS. The restored missing phase current sensors are used to calculate the $d$-axis and $q$-axis current components by applying the synchronously rotating reference frame transformation. The resulting $d q$ currents $i_{d}$ and $i_{q}$ in the three (missing) sensor tests are compared in Fig. 11. Compared to the case of no sensor missing, the values of $i_{d}$ and $i_{q}$, calculated by using the restored missing phase currents, only deviate slightly during the transient system oscillations after the grid fault. As discussed in Section IV, the unbalanced fault has no notable effect on the SERS performance since the unbalanced operation caused by the fault only exists for a very short period of time. These results indicate that the SERS correctly restores the missing current sensors during steady state as well as during the transient disturbance with a significantly reduced sampling frequency of $50 \mathrm{~Hz}$. Therefore, the SERS provides a set of fault-tolerant complete sensor data for the SSSC controllers.

Fig. 12 compares the results of G7 angular speed $\omega_{7}$ and the transmitted active power $P_{L}$ by the transmission line 23-24 for the three sensor tests. The responses of $\omega_{7}$ when two or all of the three crucial phase current sensor measurements are missing are almost the same as those in the case of no sensor missing. Compared to the synchronous generator angular speed, the active power $P_{L}$ is more sensitive to the missing-current-sensor faults because of the direct electrical relationship between the
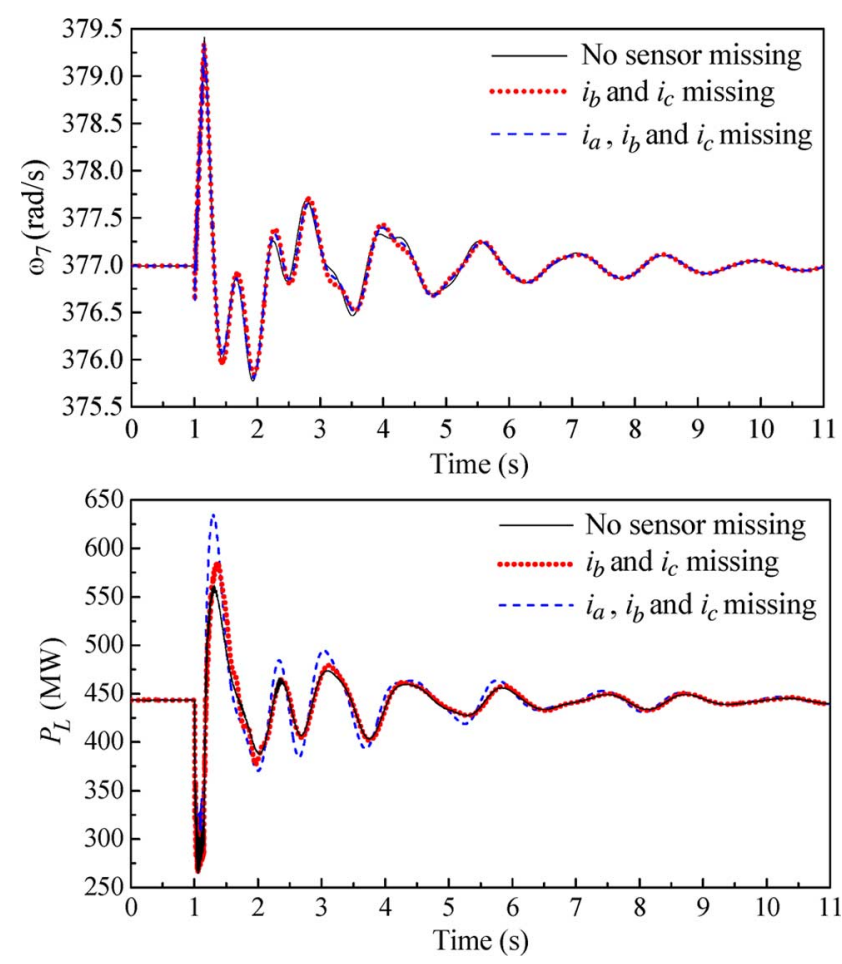

Fig. 12. Dynamic performance of the MSFTC with different (missing) sensor tests during a single-phase-to-ground (A-G) fault: $\omega_{7}$ and $P_{L}$.

active power and the currents. As a result, the damping control of the MSFTC degrades slightly but is still effective when multiple current sensors are missing, as shown in Fig. 12 for $P_{L}$. These results demonstrate that the MSFTC does provide fault-tolerant effective control for the SSSC and the power network even when multiple crucial phase current sensors are not available. In addition, neither the reduced sampling frequency at which the SERS provides the restored missing sensor data to the SSSC controllers, nor the short-term unbalanced operation caused by the fault has any notable effect on the performance of the MSFTC.

\section{B. Three-Phase-to-Ground (ABC-G) Fault}

A $150 \mathrm{~ms}$ temporary three-phase-to-ground short circuit is now applied at the bus 22 end of the transmission line 21-22 for the same three (missing) sensor tests as in the previous subsection A. Fig. 13 compares the results of $i_{d}$ and $i_{q}$ in the three (missing) sensor tests. Again, the SERS correctly restores the two and three missing current sensor data. The responses of $\omega_{7}$ and $P_{L}$ are then compared in Fig. 14. The MSFTC correctly controls the SSSC and the power system back to the prefault steadystate operating condition even when multiple crucial phase current sensors are missing. These results show that the MSFTC provides fault-tolerant effective control for the SSSC and the power network during a balanced grid fault, and that the reduced sampling frequency has no notable effect on the performance of the SERS and MSFTC.

\section{Change of Load Conditions}

The same three (missing) sensor tests as in the previous subsections are again performed. During each test, the three-phase 

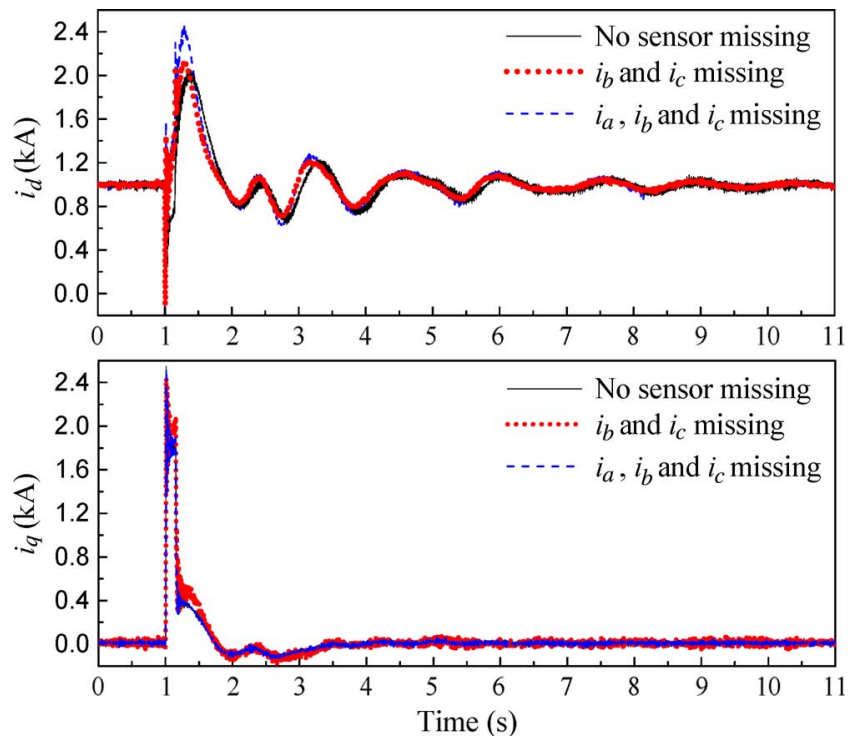

Fig. 13. Dynamic performance of the MSFTC with different (missing) sensor tests during a three-phase-to-ground (ABC-G) fault: $i_{d}$ and $i_{q}$.
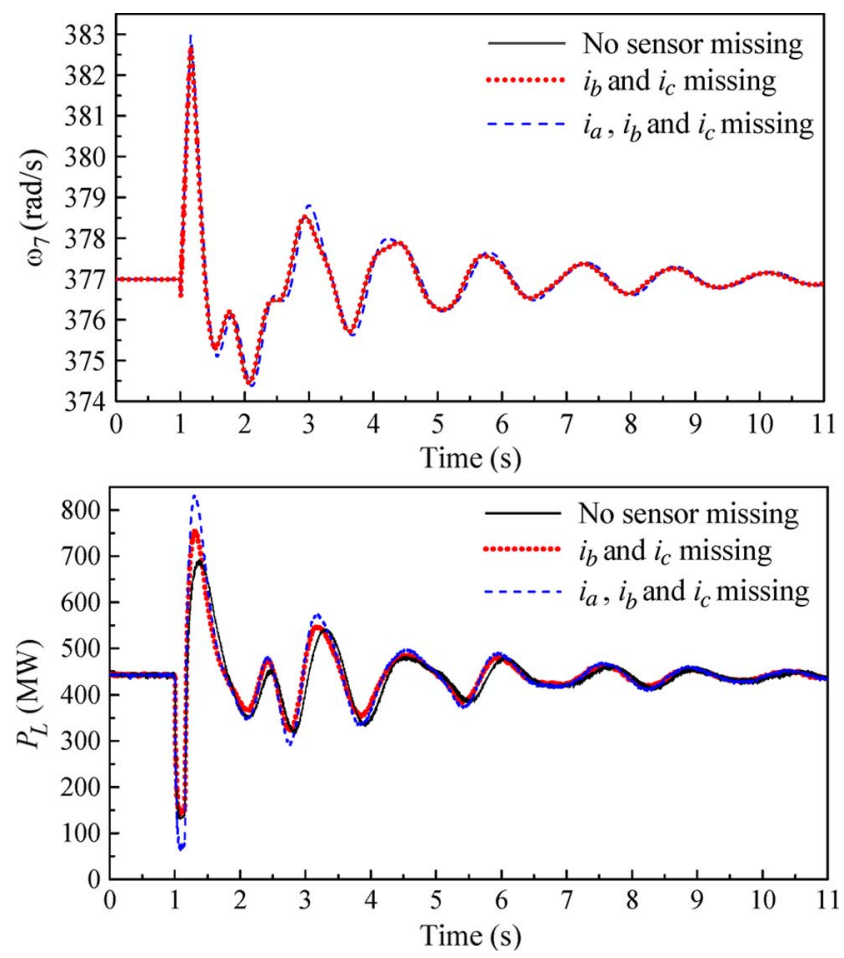

Fig. 14. Dynamic performance of the MSFTC with different (missing) sensor tests during a three-phase-to-ground (ABC-G) fault: $\omega_{7}$ and $P_{L}$.

electric load at bus 24 is increased from 308.6 MW and -92 MVAr to $608.6 \mathrm{MW}$ and -2 MVAr at $t=1 \mathrm{~s}$, respectively. After the change of the load condition, the system settles down to a new operating point. However, the auto-encoder in each MSR block of the SERS has not been trained at this new operating point since the sensors were missing before the load change.

Fig. 15 shows the results of $i_{d}$ and $i_{q}$, in the three (missing) sensor tests. The change of load condition results in a small transient in $i_{d}$ and $i_{q}$. Their steady-state values change from
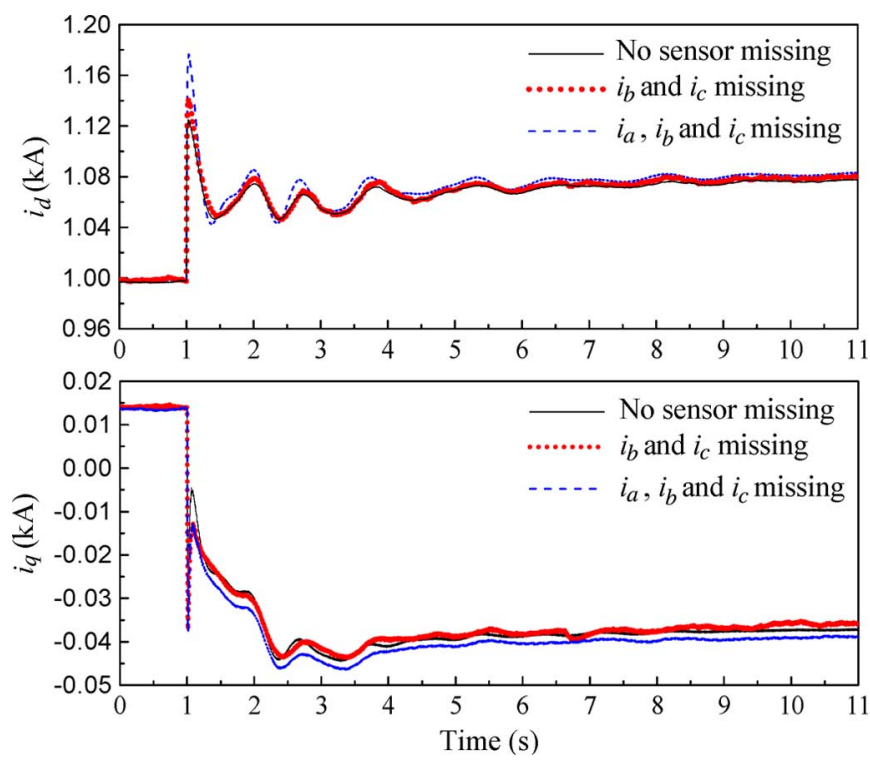

Fig. 15. Dynamic performance of the MSFTC with different (missing) sensor tests during an increase of electric load at bus 24: $i_{d}$ and $i_{q}$.

$1.0 \mathrm{kA}$ and $0.015 \mathrm{kA}$ to $1.08 \mathrm{kA}$ and $-0.04 \mathrm{kA}$, respectively. The SERS correctly restores the two and three missing current sensors when the system changes to a new operating condition.

The responses of $\omega_{7}$ and $P_{L}$ are compared in Fig. 16 . Although the dynamic performance degrades slightly when multiple crucial phase current sensors are missing, the MSFTC still correctly controls the SSSC and power network to adapt to the new operating condition after the change of load condition. These results clearly show that the desired fault-tolerance is achieved by the MSFTC.

The missing sensor tests are also performed for two other sets of sensor measurements $v_{c a}, v_{c b}, v_{c c}$, and $v_{24 a}, v_{24 b}, v_{24 c}$. Similar results as in Figs. 11-16 are obtained.

\section{Discussions ON SAMPLING FREQUENCY}

Sampling frequency is not a problem in software-based simulation studies because all of the required computation can be completed in one sampling period. In [7] and [8], the sampling frequency for MSR implementation is chosen to be $1 \mathrm{kHz}$. The resulting MSR is able to perfectly restore a $60 \mathrm{~Hz}$ sinusoidal waveform for each missing sensor data. In [9], a low sampling frequency of $50 \mathrm{~Hz}$ is used, as in this paper, and the resulting MSR still correctly restores the missing sensor data.

However, in real-time hardware implementations, sampling frequency is a critical issue for any real-time algorithm and control strategy. Generally, a high sampling frequency is expected. However, there are two contradictory inherent limits on choosing the sampling frequency: an upper limit and a lower limit.

The upper limit is placed by the computational cost of the algorithm and the available hardware. Specifically, in this paper, the upper limit is determined by the size of the auto-encoder and PSO, the convergence rate, and efficiency of the MSR, the number of missing sensor data, the performance of the DSP, $\mathrm{A} / \mathrm{D}$ and $\mathrm{D} / \mathrm{A}$ modules, and communication channels, etc. To ensure the convergence of each MSR in the SERS to restore 


\section{CONCLUSION}
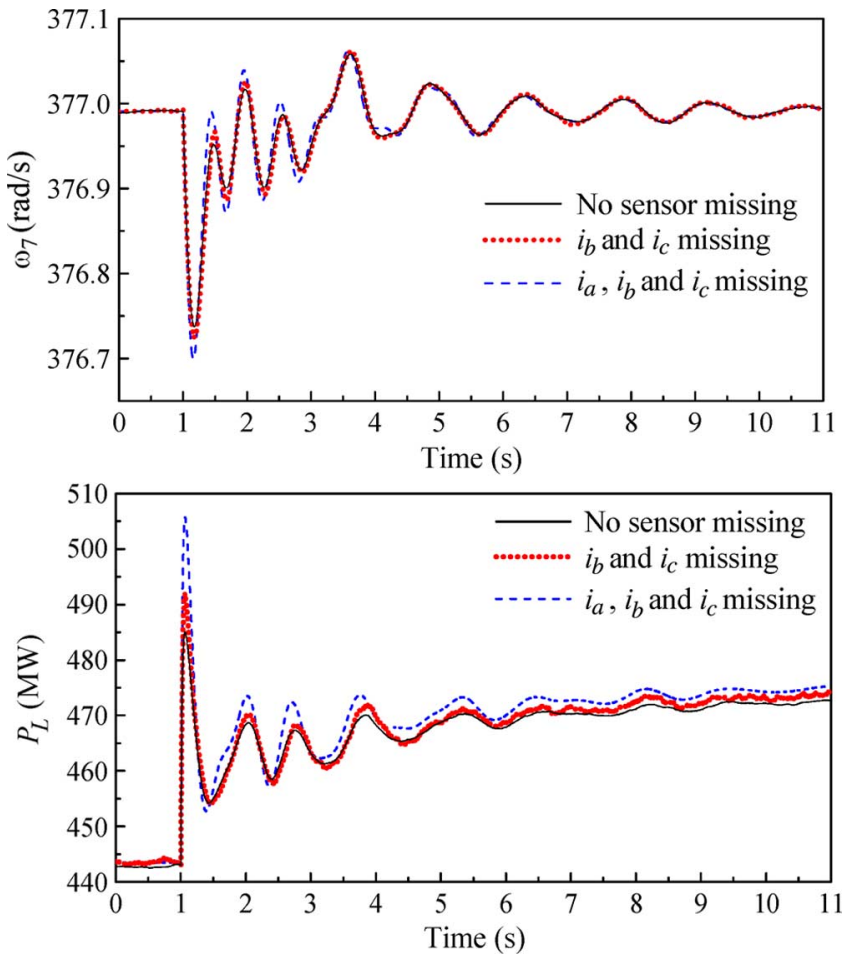

Fig. 16. Dynamic performance of the MSFTC with different (missing) sensor tests during an increase of electric load at bus 24: $\omega_{7}$ and $P_{L}$.

all three missing phase currents during each sampling period, the maximum sampling frequency is limited by $60 \mathrm{~Hz}$. Higher sampling frequencies than $60 \mathrm{~Hz}$ may result in the failure of the MSR to restore the missing data during some sampling periods; and, as a result, the performance of the SSSC controllers will degrade. A further increase of the sampling frequency, say more than $100 \mathrm{~Hz}$, results in the complete failure of the MSR and, consequently, the failure of the SSSC controllers.

The lower limit is placed by the characteristics of the system dynamics and the requirement of the SSSC controllers. A practical power system typically exhibits dynamics with frequencies less than a few Hertz. Therefore, when designing the SSSC controllers, the measured three-phase voltages and currents are normally transformed into $d q$ synchronous reference frame variables, which are all dc quantities at steady state and oscillate at frequencies less than a few Hertz during disturbances. Other variables, such as generator speed, system frequency, active and reactive power, also vary at less than a few Hertz. Therefore, the reduced sampling frequency of $50 \mathrm{~Hz}$ is sufficient for the SSSC controllers to use these low-frequency or dc variables to provide correct control actions. However, a basic rule is that the sampling frequency should be chosen at least ten times the frequency of the system dynamics in order to ensure an acceptable performance of the controllers. In this application, the frequencies of the system dynamics are around $1 \mathrm{~Hz}$. Therefore, a minimum sampling frequency of $10 \mathrm{~Hz}$ should be used. Any sampling frequency less than $10 \mathrm{~Hz}$ results in a significant degradation of the MSR performance and, consequently, a significant degradation of the SSSC controller performance.
Fault tolerance is an essential requirement for modern power system control. This paper has proposed an MSFTC strategy for controlling an SSSC connected to a power network. The MSFTC consists of a sensor evaluation and (missing sensor) restoration scheme (SERS) and a conventional internal and external SSSC control scheme (without any fault-tolerant design). The conventional control scheme provides the correct control actions for the SSSC under the condition that all of the required sensor data are available. The SERS evaluates the integrity of the time-varying sensor measurements used by the conventional SSSC controllers. If some sensors are missing, it is able to detect and restore the missing sensor data. The restored missing sensor data are then used by the SSSC controllers, which provide an MSFTC for the SSSC.

The proposed MSFTC has been validated by real-time implementations of an SSSC connected to the IEEE 10-machine 39-bus system on the RTDS and TMS320C6701 DSP platform. The SSSC and power network have been subjected to various grid disturbances and missing sensor faults (with two or three phase current sensors missing). Results have shown that the SERS correctly restores the data from multiple missing current sensors, and the resulting MSFTC provides fault-tolerant effective control for the SSSC and the power network during steady state, and transient state of unbalanced and balanced grid faults as well as a change of load conditions.

\section{REFERENCES}

[1] L. Gyugyi, C. D. Schauder, and K. K. Sen, "Static synchronous series compensator: A solid-state approach to the series compensation of transmission lines," IEEE Trans. Power Del., vol. 12, no. 1, pp. 406-417, Jan. 1997.

[2] B. S. Rigby and R. G. Harley, "An improved control scheme for a series-capacitive reactance compensator based on a voltage-source inverter," IEEE Trans. Ind. Appl., vol. 34, no. 2, pp. 355-363, Mar./Apr. 1998.

[3] B. S. Rigby, N. S. Chonco, and R. G. Harley, "Analysis of a power oscillation damping scheme using a voltage-source inverter," IEEE Trans. Ind. Appl., vol. 38, no. 4, pp. 1105-1113, Jul./Aug. 2002.

[4] W. Qiao and R. G. Harley, "Indirect adaptive external neuro-control for a series capacitive reactance compensator based on a voltage source PWM converter in damping power oscillations," IEEE Trans. Ind. Electron., vol. 54, no. 1, pp. 77-85, Feb. 2007.

[5] R. J. Patton, "Fault-tolerant control: The 1997 situation," in Proc. IFAC Symp. Fault Detection, Supervision, Safety for Technical Processes, Hull, U.K., Aug. 1997, pp. 1033-1055.

[6] O. Alsac, N. Vempati, B. Stott, and A. Monticelli, "Generalized state estimation,” IEEE Trans. Power Syst., vol. 13, no. 3, pp. 1069-1075, Aug. 1998.

[7] W. Qiao, R. G. Harley, and G. K. Venayagamoorthy, "A fault-tolerant P-Q decoupled control scheme for static synchronous series compensator," in Proc. IEEE Power Eng. Soc. General Meeting, Montreal, QC, Canada, Jun. 18-22, 2006.

[8] W. Qiao, Z. Gao, R. G. Harley, and G. K. Vanayagamoorthy, "Robust neuro-identification of nonlinear plants in electric power systems with missing sensor measurements," Eng. Appl. Artif. Intell., vol. 12, no. 4, pp. 604-618, Jun. 2008.

[9] W. Qiao, R. G. Harley, and G. K. Venayagamoorthy, "Fault-tolerant optimal neurocontrol for a static synchronous series compensator connected to a power network," IEEE Trans. Ind. Appl., vol. 44, no. 1, pp. 74-84, Jan./Feb. 2008.

[10] H. Bouland and Y. Kamp, "Auto-association by multi-layer perceptrons and singular value decomposition," Biol. Cybern., vol. 59, no. 4-5, pp. 291-294, 1988 . 
[11] M. A. Kramer, "Autoassociative neural networks," Comput. Chem. Eng., vol. 16, no. 4, pp. 313-328, 1992.

[12] J. Kennedy and R. C. Eberhart, "Particle swarm optimization," in Proc. IEEE Int. Conf. Neural Networks, Nov. 27-Dec. 1, 1995, vol. 4, pp. 1942-1948.

[13] Y. del Valle, G. K. Venayagamoorthy, S. Mohagheghi, J. C. Hernandez, and R. G. Harley, "Particle swarm optimization: Basic concepts, variants and applications in power systems," IEEE Trans. Evol. Comput., vol. 12, no. 2, pp. 171-195, Apr. 2008.

[14] B. B. Thompson, R. J. Marks, and M. A. El-Sharkawi, "On the contractive nature of autoencoders: Application to missing sensor restoration," in Proc. Int. Joint Conf. Neural Networks, Jul. 20-24, 2003, pp. 3011-3016.

[15] P. J. Werbos, "Backpropagation through time: What it does and how to do it," Proc. IEEE, vol. 78, no. 10, pp. 1550-1560, Oct. 1990.

[16] W. A. Kirk and M. A. Khamsi, An Introduction to Metric Spaces and Fixed Point Theory. New York: Wiley, 2001.

[17] F. Blaabjerg, J. K. Pedersen, U. Jaeger, and P. Thoegersen, "Single current sensor technique in the DC link of three-phase PWM-VS inverters: A review and a novel solution," IEEE Trans. Ind. Appl., vol. 33, no. 5, pp. 1241-1253, Sep./Oct. 1997.

[18] W. Qiao, W. Zhou, J. M. Aller, and R. G. Harley, "Wind speed estimation based sensorless output maximization control for a wind turbine driving a DFIG," IEEE Trans. Power Electron., vol. 23, no. 3, pp. 1156-1169, May 2008

[19] D. Reichelt, E. Ecknauer, and H. Glavitsch, "Estimation of steady-state unbalanced system conditions combining conventional power flow and fault analysis software," IEEE Trans. Power Syst., vol. 11, no. 1, pp. 422-427, Feb. 1996.

[20] T. Athay, R. Podmore, and S. Virmani, "A practical method for direct analysis of transient stability," IEEE Trans. Power App. Syst., vol. PAS-98, no. 2, pp. 573-584, Mar./Apr. 1979.

[21] M. H. Haque, "Damping improvement by FACTS devices: A comparison between STATCOM and SSSC," Elect. Power Syst. Res., vol. 76, pp. 865-872, 2006.

[22] F. L. Alvarado, J. Meng, C. L. DeMarco, and W. S. Mota, "Stability analysis of interconnected power systems coupled with market dynamics," IEEE Trans. Power Syst., vol. 16, no. 4, pp. 695-701, Nov. 2001.

[23] P. Forsyth, T. Maguire, and R. Kuffel, "Real time digital simulation for control and protection system testing," in Proc. 35th Annu. IEEE Power Electronics Specialists Conf., Jun. 20-25, 2004, pp. 329-335.

[24] “OMNIBUS User's Manual,” Innovative Integration, Simi Valley, CA, Feb. 2001

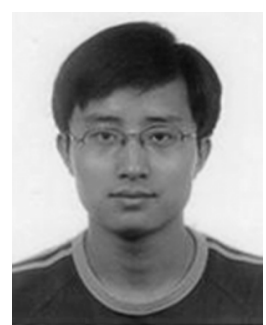

Wei Qiao (S'05-M'08) received the B.Eng. and M.Eng. degrees in electrical engineering from Zhejiang University, Hangzhou, China, in 1997 and 2002 , respectively, the M.S. degree in high-performance computation for engineered systems from the Singapore-MIT Alliance (SMA), Singapore, in 2003, and the Ph.D. degree in electrical engineering from the Georgia Institute of Technology, Atlanta, in 2008.

From 1997 to 1999, he was an Electrical Engineer with China Petroleum \& Chemical Corporation (Sinopec). Currently, he is an Assistant Professor of Electrical Engineering at the University of Nebraska-Lincoln. His research interests include renewable energy systems and distributed generation, microgrids, power system control, stability and performance optimization, power electronics, electric machines, flexible ac transmission system devices, and the application of computational intelligence in electric power and energy systems. He is the first author of two book chapters, 11 refereed journal papers, and more than 20 refereed international conference proceeding papers.

Dr. Qiao is the Technical Program Co-Chair of the 2009 IEEE Symposium on Power Electronics and Machines in Wind Applications. He was the recipient of the first price in the Student Paper and Poster Competition of the IEEE Power Engineering Society General Meeting 2006 in Montreal, QC, Canada.

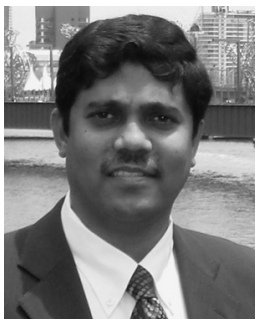

Ganesh Kumar Venayagamoorthy (S'91-M'97-SM'02) received the Ph.D. degree in electrical engineering from the University of KwaZulu Natal, Durban, South Africa, in 2002.

Currently, he is an Associate Professor of Electrical and Computer Engineering and the Director of the Real-Time Power and Intelligent Systems Laboratory at the Missouri University of Science and Technology (Missouri S\&T), Rolla. He was a Visiting Researcher with ABB Corporate Research, Vasteras, Sweden, in 2007. His research interests are the development and applications of computational intelligence for power systems stability and control, alternative sources of energy, and FACTS devices. He has published two edited books, five book chapters, 57 refereed journal papers, and more than 200 refereed international conference proceeding papers. He has attracted in excess of U.S.\$4 million in competitive research funding from external funding agencies.

Dr. Venayagamoorthy is the Chair of the Working Group on Intelligent Control Systems and the Secretary of the Intelligent Systems subcommittee of the IEEE Power Engineering Society. He is the General Chair of 2008 IEEE Swarm Intelligence Symposium and the Program Chair of the 2009 IEEE-INNS International Joint Conference on Neural Networks. He was a recipient of the 2007 U.S. Office of Naval Research Young Investigator Program Award, the 2004 National Science Foundation CAREER Award, the 2006 IEEE Power Engineering Society Walter Fee Outstanding Young Engineer Award, the 2005 IEEE Industry Applications Society (IAS) Outstanding Young Member Award, the 2003 INNS Young Investigator Award, and a 2007/2005 Missouri S\&T Faculty Excellence Award.

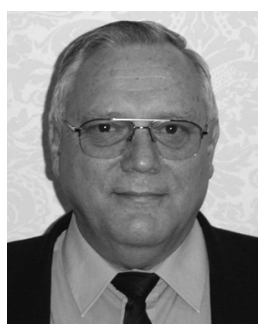

Ronald G. Harley (M'77-SM'86-F'92) received the M.Sc.Eng. degree (Hons.) in electrical engineering from the University of Pretoria, Pretoria, South Africa, in 1965, and the Ph.D. degree in electrical engineering from London University, London, U.K., in 1969.

In 1971, he was appointed Chair of Electrical Machines and Power Systems at the University of Natal in Durban, Durban, South Africa. Currently, he is the Duke Power Company Distinguished Professor at the Georgia Institute of Technology, Atlanta. His research interests include the dynamic behavior and condition monitoring of electric machines, motor drives, power systems and their components, and controlling them with the use of power electronics and intelligent control algorithms. He has coauthored approximately 400 papers in refereed journals and international conferences and three patents. During 2000 and 2001, he was one of the IEEE Industry Applications Society's six Distinguished Lecturers. In 2005, he received The Cyril Veinott Electromechanical Energy Conversion Award from the IEEE Power Engineering Society for "Outstanding contributions to the field of electromechanical energy conversion." 